Check for updates

Cite this: J. Anal. At. Spectrom., 2019, 34,1233

Received 3rd March 2019 Accepted 11th April 2019 DOI: 10.1039/c9ja00075e rsc.li/jaas

\section{Glow discharge optical emission spectrometry for quantitative depth profiling of CIGS thin-films $\uparrow$}

Tim Kodalle, (D)*a Dieter Greiner, ${ }^{a}$ Varvara Brackmann, ${ }^{\text {tb }}$ Karsten Prietzel, ${ }^{a}$ Anja Scheu, ${ }^{a}$ Tobias Bertram, ${ }^{a}$ Pablo Reyes-Figueroa, ${ }^{a}$ Thomas Unold, ${ }^{a}$ Daniel AbouRas, ${ }^{a}$ Rutger Schlatmann, ${ }^{\text {ac }}$ Christian A. Kaufmann ${ }^{a}$ and Volker Hoffmann ${ }^{b}$

Determining elemental distributions dependent on the thickness of a sample is of utmost importance for process optimization in different fields e.g. from quality control in the steel industry to controlling doping profiles in semiconductor labs. Glow discharge optical emission spectrometry (GD-OES) is a widely used tool for fast measurements of depth profiles. In order to be able to draw profound conclusions from GD-OES profiles, one has to optimize the measurement conditions for the given application as well as to ensure the suitability of the used emission lines. Furthermore a quantification algorithm has to be implemented to convert the measured properties (intensity of the emission lines versus sputtering time) to more useful parameters, e.g. the molar fractions versus sample depth (depth profiles). In this contribution a typical optimization procedure of the sputtering parameters is adapted to the case of polycrystalline $\mathrm{Cu}(\mathrm{In}, \mathrm{Ga})(\mathrm{S}, \mathrm{Se})_{2}$ thin films, which are used as absorber layers in solar cell devices, for the first time. All emission lines used are shown to be suitable for the quantification of the depth profiles and a quantification routine based on the assumption of constant emission yield is used. The accuracy of this quantification method is demonstrated on the basis of several examples. The bandgap energy profile of the compound semiconductor, as determined by the elemental distributions, is compared to optical measurements. The depth profiles of $\mathrm{Na}$ - the main dopant in these compounds - are correlated with measurements of the open-circuit voltage of the corresponding devices, and the quantification of the sample depth is validated by comparison with profilometry and X-ray fluorescence measurements.

\section{Introduction}

Solar cells based on $\mathrm{Cu}(\mathrm{In}, \mathrm{Ga})(\mathrm{Se}, \mathrm{S})_{2}$ (CIGSSe) absorber layers exhibit the highest power conversion efficiencies (PCE) of all polycrystalline thin film technologies, ${ }^{1}$ which makes this type of solar cell a promising candidate for managing the challenge of clean electric energy supply in the future. To achieve PCEs as high as the current world record of $22.9 \%,{ }^{2}$ it is crucial to adjust the bandgap energy $\left(E_{\mathrm{g}}\right)$ profile and the gradients of the quasifermi levels within the CIGSSe absorber layer. ${ }^{2-4}$ To do so, one has to adjust the in-depth elemental gradient of In and Ga as well as Se and $\mathrm{S}$ in the compound semiconductor carefully. ${ }^{5}$ Typically a double-graded $E_{\mathrm{g}}$-structure is used to achieve the

\footnotetext{
${ }^{a}$ Helmholtz-Zentrum Berlin für Materialien und Energie GmbH, Hahn-Meitner Platz 1, D-14109, Berlin, Germany. E-mail: tim.kodalle@helmholtz-berlin.de

${ }^{b}$ IFW Dresden, Institute for Complex Materials, Helmholtzstraße 20, D-01069 Dresden, Germany

'Hochschule für Technik und Wirtschaft Berlin, Treskowallee 8, 10318 Berlin, Germany $\dagger$ Electronic supplementary information (ESI) available. See DOI: 10.1039/c9ja00075e

¥ Present Address: Fraunhofer Institute for Photonic Microsystems IPMS - Center Nanoelectronic Technologies CNT, Königsbrücker Straße 178, D-01099 Dresden, Germany.
}

highest PCEs. The minimum $E_{\mathrm{g}}$ is adapted to the spectrum of the sunlight according to the Shockley-Queisser-limit, ${ }^{6}$ while $E_{\mathrm{g}}$ increases towards the interfaces at the front- and the backcontact. By achieving a higher $E_{g}$ at these interfaces, the recombination velocity there can be reduced., ${ }^{7,8}$ Furthermore one can optimize the offset of the conduction bands of the absorber layer and the neighbouring buffer layer at the front interface. Depending on the deposition process such an $E_{g^{-}}$ profile can be achieved by adjusting the ratios of the molar fraction of $\mathrm{Ga}$ and In $\left(\chi_{\mathrm{Ga}} /\left(\chi_{\mathrm{Ga}}+\chi_{\mathrm{In}}\right)=\mathrm{GGI}\right)$ within a S-free $\mathrm{Cu}(\mathrm{In}, \mathrm{Ga}) \mathrm{Se}_{2}$ (CIGSe) absorber layer. ${ }^{9}$ But when using e.g. a sequential deposition process based on a rapid thermal processing (RTP-) step of metal precursors in a chalcogen atmosphere, there is no direct control of the GGI and one needs to adjust the $E_{\mathrm{g}}$-gradient towards the front interface by the additional incorporation of S into CIGSe. Therefore one has to adjust the molar fractions of the anions accordingly $\left(\chi_{\mathrm{S}} /\left(\chi_{\mathrm{S}}+\chi_{\mathrm{Se}}\right)=\right.$ SSSe). ${ }^{8}$

Pulsed glow discharge optical emission spectroscopy (GDOES) is a fast and well-suited technique to measure the elemental distribution with sufficient depth resolution in the 2-3 $\mu \mathrm{m}$ thick CIG(S)Se layers. ${ }^{10}$ Although GD-OES cannot achieve similar limits of detection to its counterparts based on mass spectrometry (MS), 
it is sensitive enough to obtain sufficiently good depth profiles of the main components of CIGSSe as well as of the main dopants. Additionally GD-OES is faster, cheaper and easier to perform than for example glow discharge mass spectrometry (GD-MS) or secondary ion mass spectrometry (SIMS), mainly because it does not require ultrahigh vacuum. Mild surface sputtering and fast sampling time of commercial light detection systems therefore make GD-OES a competitive and fast method for depth profiling of thin layers (few $\mathrm{nm}$ to more than $100 \mu \mathrm{m}$ ). For a more comprehensive comparison of GD-OES with various other depth profiling techniques the reader is referred to a previous study published by Abou-Ras et al. ${ }^{\mathbf{1 0}}$

Since the measured raw data of a GD-OES depth profile (intensity $v s$. sputtering time) are not directly suitable for an accurate analysis of $e . g$. the $E_{\mathrm{g}}$-profile, a reliable quantification procedure is required to obtain the molar fractions of the contained elements as a function of the thickness of the absorber layer. Although GD-OES is already widely used for obtaining depth profiles of CIGSSe thin films, ${ }^{11-18}$ to the best of our knowledge there is no study published yet to test its overall applicability and the specific suitability of the used atomic transitions of the elements contained in CIGS for this purpose.

In the present contribution we demonstrate how to optimize the glow discharge conditions for accurate measurements of CIGSSe thin films and present a quantification algorithm based on the main assumption of constant emission yields adapted to these types of samples. ${ }^{19}$ Furthermore we test the used emission lines for their usability in this quantification and evaluate the resulting quantified depth profiles for several applications.

\section{Experimental}

\section{Sample preparation}

The CIGSe-layers used as reference samples are deposited on soda-lime glass covered by $800 \mathrm{~nm}$ Mo. The CIGSe layer is grown by single stage co-evaporation of the contained elements onto the heated substrates in a vacuum based deposition process. Details of the utilized setup can be found in ref. 20. The CuInS sample used for calibration was deposited in a similar process using a different system. ${ }^{21}$ A cold-pressed CIGSSe:Na-reference sample was used for the quantification of $\mathrm{S}$ and Na. The cylindrical sample with a diameter and a thickness of $2 \mathrm{~cm}$ each was provided by Evochem Advanced Materials GmbH. For calibration purposes, certified cast $\mathrm{Cu}: \mathrm{S}$ samples as well as sintered $\mathrm{Cu}: \mathrm{NaCl}$ were used. The sintering process is described in ref. 22 . The properties of all reference and calibration samples used in this study are summarized in the ESI. $\dagger$ The samples used for the verification of the method (solar cell applications) are either grown in the same system as the CIGSe reference layers, but using a three-stage process ( $\mathrm{S}$-free samples $)^{\mathbf{2 0}}$ or are grown using a sequential RTP (in the case of CIGSSe-samples). Details of this RTP are published in ref. 23.

\section{Measurement techniques}

All GD-OES depth profiles were measured using a GDA650system built by Spectruma Analytik in pulsed radio frequency (rf) mode, in order to be able to sputter the semiconducting absorber layer and to avoid heat-induced damage of the glass substrate. The system uses an Ar-plasma for sputtering and a CCD-array to detect the photons emitted during the relaxation of the atoms and ions, which were detached from the matrix and excited in the plasma. For the rf mode, the system is equipped with a Grimm-type glow discharge source including an anode with an inner diameter of $2.5 \mathrm{~mm}$ and a non-conducting cathode plate with front end coupling. ${ }^{24}$ Details of the setup can be found in ref. 25 and 26.

Profiles of the sputtered craters are measured using a Dektak and an FRT MicroProf profilometer. The current densityvoltage $(j-V)$ measurements were performed under standard test conditions (AM $1.5 \mathrm{~g}$ spectrum, $1000 \mathrm{~W} \mathrm{~m}^{-2}, 25{ }^{\circ} \mathrm{C}$ ) using a WACOM A+ solar simulator; external quantum efficiency (EQE)-measurements were carried out using a self-built setup, and X-ray fluorescence spectrometry (XRF) measurements were performed with a Rigaku ZSX Primus II.

\section{Results and discussion}

\section{Optimization of the sputtering parameters for flat crater profiles}

The depth resolution of GD-OES is limited by the formation of a nonplanar sputtered crater in the sample and sputter induced roughening of the sample surface.

To reach the best possible depth resolution and to measure a reliable depth profile of the sample by means of GD-OES, it is necessary to optimize the analysis conditions, i.e. to reduce the curvature and roughness in the sputtered crater to a minimum. Hence, it is necessary to adjust the parameters of the sputtering cell accordingly, which are the voltage $U$, the current $I$, the pressure $p$, and in case a pulsed mode is used also the pulse length $l$, the frequency $f$, and the duty cycle.

The $U, I$ and $p$, as well as duty cycle, $l$, and $f$ are interdependent. To simplify the optimization work, it was assumed that $p$ and $f$ have a smaller effect on the shape of the sputtered crater as compared with the other parameters. ${ }^{25,27,28}$ However, in the case of rf discharge, it is hard to measure the current reliably. Therefore, in the present work the pressure is measured instead. Thus, there are four parameters to optimize: $U, p, l$, and the duty cycle. For a good understanding of the influence of each parameter separately, it is important to vary just one parameter at a time and keep all others constant.

Voltage and pressure. In a previous study it was shown that high voltages and high currents (or pressures) lead to a convex crater shape, whereas low values of these parameters lead to a concave one. ${ }^{26}$ In order to achieve a flat crater profile, moderate voltage and pressure were chosen in the present study.

Duty cycle. Variation of the duty cycle at constant pulse length does not lead to any change of the crater shape. ${ }^{29}$ However, higher duty cycles mean more power applied to the sample surface, and hence, enhanced heat introduction. This heat can lead to sputter-induced surface roughening of the CIGS samples caused presumably by $\mathrm{Cu}$ agglomeration. ${ }^{30}$ Therefore duty cycles lower than $10 \%$ were used. 
Pulse length. The pulse length is a crucial parameter for the crater shape. Pulses which are shorter than 50-150 $\mu$ s cause convex crater shapes. ${ }^{29}$ Furthermore, in the case of rf discharge the application of short pulses suppresses the sputtering substantially. At pulses of 10 or $20 \mu \mathrm{s}$ nearly no sputtering occurs. ${ }^{25,29}$ Accordingly, pulses around $100 \mu$ s were used in the present study.

By carefully optimizing all parameters according to these rules, the optimal analysis parameters for the CIGSe samples were chosen to be: $p=2.5 \mathrm{hPa}, U=650 \mathrm{~V}, f=451 \mathrm{~Hz}, l=89 \mu \mathrm{s}$, duty cycle: $4 \%$. Using these parameters it takes about 5 min to sputter through one of our standard CIGSe-layers with a thickness of approx. $2.5 \mu \mathrm{m}$. In Fig. 1 the profile of a sputtered crater generated with these parameters and measured using the profilometer is shown. Some examples of how the profile of the sputtered crater is influenced by non-optimal sputtering parameters are shown in the ESI (Fig. SI1 $\dagger$ ).

Please note that the sputtering parameters depend on the experimental setup and should be established for each system individually, according to the aforesaid principles.

\section{Quantification of the GD-OES depth profiles}

The aim of the quantification of the GD-OES profiles is to derive profiles of the molar fraction of each element $v s$. the layer depth from the measured intensity $v s$. sputtering time.

To do so an algorithm based on the assumption of constant emission yield is used. ${ }^{19}$ The emission yield $R_{\mathrm{j}}$ for atoms of element $\mathrm{j}$ is defined as the number of photons emitted per atom of this element removed from the sample's surface. $R_{\mathrm{j}}$ is one of the proportionality factors between the measured intensity $I_{\mathrm{j}}$ of element $\mathrm{j}$ - or more precisely the intensity of a chosen emission line transition of this element - and the actual mass fraction $c_{\mathrm{j}}$ of this element in the matrix. The other proportionality factors are the sputtering rate $q$ and the instrumental detection efficiency $k_{\mathrm{j}}$. The factor $S_{\mathrm{j}}$ takes selfabsorption into account and becomes a function of $c_{\mathrm{j}} q$, which

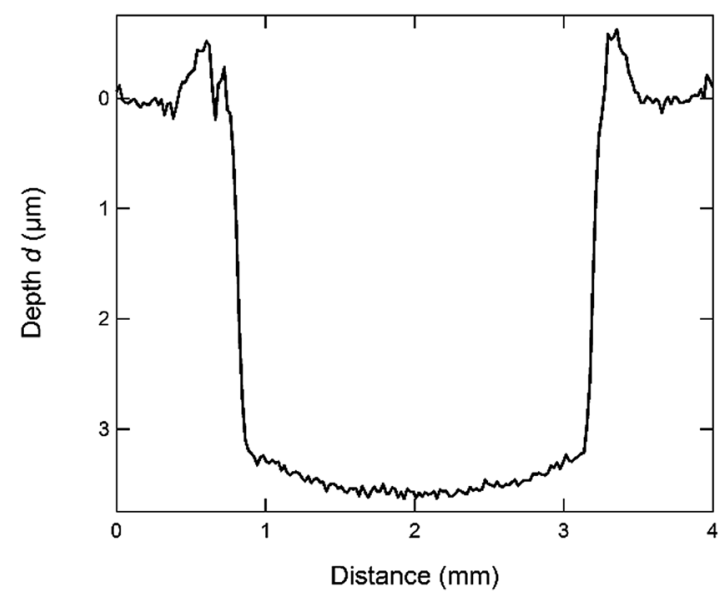

Fig. 1 Profilometer scan of a sputtered crater in a Mo/CIGSe layer stack. The image shows an average of 21 line scans taken within a distance of $\sim 0.4 \mathrm{~mm}$ in the middle of the file; moderate voltage and pressure were chosen in the present study. is smaller than 1, if emitted photons are absorbed after emission. Additionally $I_{\mathrm{j}}$ depends on a background value $b_{\mathrm{j}}$ determined by the used instrument: ${ }^{27}$

$$
I_{\mathrm{j}}=k_{\mathrm{j}} S_{\mathrm{j}} R_{\mathrm{j}} c_{\mathrm{j}} q+b_{\mathrm{j}}
$$

By measuring the intensities of reference samples with known $c_{\mathrm{j}}$ and $q$, one can transform measured intensities from unknown samples into their mass fractions using eqn (1). To do so one has to account for the background of the given emission line e.g. by choosing lines with a background that is small compared to the actual intensities or by measuring and subtracting $b_{\mathrm{j}}$. Secondly one has to determine whether the intensity of the used emission line is linearly dependent on the introduced mass of element $\mathrm{j}$ per time in the plasma $c_{\mathrm{j}} q$. This information is obtained by creating calibration curves for all used emission lines. In these calibration curves usually the intensity is plotted versus the product $c_{\mathrm{j}} q$. At the intersection of these curves and the intensity-axis one can determine $b_{\mathrm{j}}$. If the curves show a linear dependence of $I_{\mathrm{j}}$ on $c_{\mathrm{j}} q$, self-absorption can be neglected and $S_{\mathrm{j}}$ becomes 1 .

Furthermore it is assumed that $k_{\mathrm{j}}$ is independent of the measured sample and the sputtering parameters, but is a constant only dependent on the utilized spectrometer. For all used emission lines this instrumental factor is included in $R_{\mathrm{j}}$. Therefore, for the calculation of $c_{\mathrm{j}} q$ of unknown samples from the measured line intensities, eqn (1) is actually used in a simplified form, if calibration shows linearity in the region of interest

$$
I_{\mathrm{j}}=R_{\mathrm{j}} c_{\mathrm{j}} q+b_{\mathrm{j}}
$$

or

$$
I_{\mathrm{j}}=R_{\mathrm{j}} c_{\mathrm{j}} q
$$

respectively in the case of a negligible background. It will be shown in the section "Calibration curves" below that it is valid to use eqn (3) in our quantification algorithm.

Determination of the emission yields. Usually, $R_{\mathrm{j}}$ is calculated from the slope of the calibration curves. However, the validity of eqn (3) allows a simple calibration using one reference sample only. The $R_{\mathrm{j}}$ can be derived from a reference sample (RS) with known concentration $c_{\mathrm{j}}^{\mathrm{RS}}$ :

$$
R_{\mathrm{j}}=\frac{I_{\mathrm{j}}^{\mathrm{RS}}}{c_{\mathrm{j}}^{\mathrm{RS}} q^{\mathrm{RS}}}=\frac{I_{\mathrm{j}}^{\mathrm{RS}}}{c_{\mathrm{j}}^{\mathrm{RS}} \frac{\sum_{\mathrm{j}} \Delta m_{\mathrm{j}}^{\mathrm{RS}}}{\Delta t}} .
$$

Here we used the sputtering rate given by the fraction of the sputtered mass of all contained elements $\Delta \sum_{\mathrm{j}} m_{\mathrm{j}}$ per sputtering time $\Delta t$. When reference samples with a homogeneous distribution of all elements within the layer are used, one can integrate over all sputtering times $\Delta t$ and conclude for the whole layer: 


$$
R_{\mathrm{j}}=\frac{I_{\mathrm{j}}^{\mathrm{RS}} t^{\mathrm{RS}}}{c_{\mathrm{j}}^{\mathrm{RS}} \sum_{\mathrm{j}} m_{\mathrm{j}}^{\mathrm{RS}}}=\frac{I_{\mathrm{j}}^{\mathrm{RS}} t^{\mathrm{RS}}}{c_{\mathrm{j}}^{\mathrm{RS}} \rho^{\mathrm{RS}} d^{\mathrm{RS}} A^{\mathrm{RS}}} .
$$

Since it is difficult to measure the sputtered mass with high accuracy, one has to use the product of the sample density $\rho$ and its volume $V$, which in the case of a reference layer is given by the thickness $d$ of the sputtered layer and the area of the sputtered crater $A$. For bulk calibration materials the density also has to be known and the sputtered mass can then be calculated from the measured crater volume. ${ }^{31}$

Quantification of the elemental concentrations. Since the emission yields are only dependent on the selected emission line of an element and the utilized spectrometer, they can be directly used to determine the mass fraction of each element of an unknown sample $c_{\mathrm{j}}^{\mathrm{u}}$ from the measured GD-OES intensity $I_{\mathrm{j}}^{\mathrm{u}}$ using eqn (3):

$$
c_{\mathrm{j}}^{\mathrm{u}}=\frac{I_{\mathrm{j}}^{\mathrm{u}}}{R_{\mathrm{j}} q} .
$$

As long as the matrix of the unknown sample is the same as the matrix of the reference sample the sputtering rate determined from the reference sample can also be used for the quantification of the unknown sample. However, in reality the unknown measurement samples commonly show different concentrations of the contained elements as well as gradients of these over the sample depth. Therefore in most cases the sputtering rate has to be determined for each sputtering cycle of the measurement of the unknown sample. To do so, it is assumed that the sum of the mass concentration as well as the molar fractions of all measured elements equals 1 (for this reason it is important to note that the quantification can only be performed, if nearly $100 \%$ of the elements present are determined):

$$
\begin{gathered}
\sum_{\mathrm{j}} \chi_{\mathrm{j}}^{\mathrm{u}}=\sum_{\mathrm{j}} c_{\mathrm{j}}^{\mathrm{u}}=1 \\
\Rightarrow q=q \sum_{\mathrm{j}} c_{\mathrm{j}}^{\mathrm{u}}=\sum_{\mathrm{j}} \frac{I_{\mathrm{j}}^{\mathrm{u}}}{R_{\mathrm{j}}} \\
\Rightarrow c_{\mathrm{j}}^{\mathrm{u}}=\frac{I_{\mathrm{j}}^{\mathrm{u}}}{R_{\mathrm{j}} q}=\frac{I_{\mathrm{j}}^{\mathrm{u}} / R_{\mathrm{j}}}{\sum_{\mathrm{j}}\left(I_{\mathrm{j}}^{\mathrm{u}} / R_{\mathrm{j}}\right)} .
\end{gathered}
$$

This can be done for each measurement step, i.e. for each time slice of the measurement. In a last step the molar fraction of each element and time slice can be calculated from the mass fraction using the atomic mass of each element $\mathrm{j}\left(M_{\mathrm{j}}\right)$ :

$$
\Rightarrow \chi_{\mathrm{j}}^{\mathrm{u}}=\frac{c_{\mathrm{j}}^{\mathrm{u}} / M_{\mathrm{j}}}{\sum_{\mathrm{j}}\left(c_{\mathrm{j}}^{\mathrm{u}} / M_{\mathrm{j}}\right)} .
$$

Calculation of the layer depth. There are two ways to calculate the depth of the sputtered crater from the sputtering time. Both will be explained theoretically here, and experimentally compared below.

In the first approach it is assumed that each layer, which is sputtered from the sample during one sputtering cycle, consists of the sum of hypothetical layers of the contained elements, e.g. a CIGSe-layer is replaced by a stack of $\mathrm{Cu}$, In, $\mathrm{Ga}$ and Se. With that assumption one can calculate the thickness of this layer by determining the hypothetical layer thickness for each element $\mathrm{j}$, which is sputtered from the sample during each sputtering cycle $\Delta t$ :

$$
\Delta z_{\mathrm{j}}=\frac{\Delta V_{\mathrm{j}}}{A}=\frac{\Delta m_{\mathrm{j}}}{A \rho_{\mathrm{j}}}=\frac{\Delta t I_{\mathrm{j}}}{A \rho_{\mathrm{j}} R_{\mathrm{j}}} \text { using } \frac{\Delta m_{\mathrm{j}}}{\Delta t}=c_{\mathrm{j}} q=\frac{I_{\mathrm{j}}}{R_{\mathrm{j}}} .
$$

This estimation uses the densities $\rho_{\mathrm{j}}$ of the pure elements. The actual sputtering depth can then be derived by summing up the individual layer thicknesses of all elements:

$$
\Delta z=\sum_{\mathrm{j}} \Delta z_{\mathrm{j}}
$$

This assumption is valid for metals and alloys, ${ }^{28}$ but can lead to some errors in other materials. As an example the densities for $\mathrm{C}$ in graphite $\left(2.26 \mathrm{~g} \mathrm{~cm}^{-3}\right)$ and diamond $\left(3.52 \mathrm{~g} \mathrm{~cm}^{-3}\right)$ differ remarkably, which shows that the use of just one density of $\mathrm{C}$ can lead to substantial errors.

Another approach therefore is to replace the elemental densities with the actual density of the sputtered layer, e.g. the CIGSe with the exact composition during that sputtering cycle:

$$
\Delta z=\frac{1}{\rho} \sum_{\mathrm{j}} \frac{\Delta t I_{\mathrm{j}}}{A R_{\mathrm{j}}}
$$

For each sputtering cycle the density of the CIGSe can be calculated by the following formula based on Vegard's law, ${ }^{32}$ assuming a constant $\mathrm{Cu}$-content:

$$
\begin{aligned}
\rho & =\frac{m_{\mathrm{UC}}}{V_{\mathrm{UC}}}=\frac{\sum_{\mathrm{j}} M_{\mathrm{j}} n_{\mathrm{j}}}{a_{\mathrm{CIGSe}}{ }^{2} c_{\mathrm{CIGSe}}} \\
& =\frac{\sum_{\mathrm{j}} M_{\mathrm{j}} n_{\mathrm{j}}}{\left((1-\mathrm{GGI}) a_{\mathrm{CISe}}+\mathrm{GGI} \times a_{\mathrm{CGSe}}\right)^{2}\left((1-\mathrm{GGI}) c_{\mathrm{CISe}}+\mathrm{GGI} \times c_{\mathrm{CGSe}}\right)} .
\end{aligned}
$$

Here, the index "UC" marks properties of the unit cell. Furthermore $n_{\mathrm{j}}$ is the number of atoms of element $\mathrm{j}$ per unit cell, and $a$ and $c$ are the lattice parameters of the chalcopyrite's unit cell. According to Vegard's law the lattice parameters of the CIGSe $(a, c)_{\text {CIGSe }}$ can be calculated using the lattice parameters of the two constituents $\mathrm{CuInSe}_{2}(a, c)_{\mathrm{CISe}}$ and $\mathrm{CuGaSe}_{2}(a, c)_{\mathrm{CGSe}}$ weighted by the GGI. ${ }^{32}$

Additionally the lattice parameters are dependent on the CGI $\left(\mathrm{CGI}=\chi_{\mathrm{Cu}} /\left(\chi_{\mathrm{Ga}}+\chi_{\mathrm{In}}\right)\right)$, but since this dependency is mostly determined by the formation of $\mathrm{Cu}$-related defects in the crystal 
structure, there is no generally valid expression derived yet. ${ }^{33,34}$ However, for a given CGI the lattice parameters can be derived experimentally - and do not depend on CGI strongly ${ }^{33,34}$ in the range usually used for highly efficient CIGSSe solar cells $0.85<$ CGI < 0.95). ${ }^{13}$ Stephan et al. ${ }^{34}$ showed that the volume of the unit cell of CISe within that CGI-range is altered by not more than $0.07 \%$. However, one has to keep in mind that the error might become relevant at very low CGI.

In Table 1 the layer thickness of seven standard CIGSe layers (with Ga-grading, $c f$. examples section) as determined using a profilometer $\left(d_{\text {Dektak }}\right)$ is shown and the deviation of the thickness from that value determined by XRF $\left(d_{\mathrm{XRF}}\right)$ and GDOES using both methods described above is displayed. In the latter measurements the depth at which the Mo-signal reaches $\chi_{\text {Mo }}=0.1$ was chosen as the end of the CIGSe-layer. In all cases the thickness, which was determined using eqn (13) and (14), i.e. $\Delta d_{\mathrm{GD}-\mathrm{CES}}^{\mathrm{CIGS}}$, is slightly higher than the one determined following eqn (11) and (12), i.e. $\Delta d_{\text {GD-OES }}^{\text {elemen }}$. All three methods show sufficiently small deviation in the range of the error of the profilometer itself confirming that the approach of combining the elemental densities is valid for CIGSe thin films.

\section{Calibration curves}

In order to obtain optimum $E_{\mathrm{g}}$-gradients, the composition of the CIGSSe can be changed drastically within one absorber layer. In standard high efficiency CIGSe solar cells ( $\mathrm{S}$ free) GGI ranges from about GGI $\approx 0.2$ at the minimum $E_{\mathrm{g}}$ up to values of $\mathrm{GGI} \approx 0.6$ at the rear interface CIGSe/Mo. Similar - though not as pronounced - changes of the concentration of $\mathrm{Cu}$ and $\mathrm{Se}$ occur near the front interface. Furthermore the concentration of $\mathrm{S}$ in CIGSSe samples usually ranges from SSSe $\approx 0.1$ to SSSe $\approx$ 0.5 , while the molar fraction of Na lies in the order of magnitude of $\chi_{\mathrm{Na}}=1 \times 10^{-4}$.

In order to be able to apply the quantification routine to these layers, one has to ensure two things:

(1) Self-absorption $S_{\mathrm{j}}$ can be neglected.

(2) The background $b_{\mathrm{j}}$ is either negligible or is measured and subtracted from the measured intensity in an additional step prior to the described quantification.

Only then the emission yield determined at one given concentration of each element can be used to quantify the

Table 1 Comparison of the thickness evaluation by XRF, DektakProfilometry and GD-OES

\begin{tabular}{lcccc}
\hline ID & $d_{\text {Dektak }}(\mu \mathrm{m})$ & $\Delta d_{\mathrm{XRF}}$ & $\Delta d_{\mathrm{GD}-\mathrm{OES}}^{\text {elementa }}$ & $\Delta d_{\mathrm{GD}-\mathrm{OES}}^{\mathrm{CIGS}}$ \\
\hline 1 & $2.22 \pm 0.06$ & $6.2 \%$ & $1.6 \%$ & $4.5 \%$ \\
2 & $2.28 \pm 0.03$ & $3.5 \%$ & $-0.9 \%$ & $2.2 \%$ \\
3 & $2.30 \pm 0.05$ & $5.6 \%$ & $-2.30 \%$ & $0.9 \%$ \\
4 & $2.38 \pm 0.02$ & $1.6 \%$ & $-1.1 \%$ & $1.1 \%$ \\
5 & $2.40 \pm 0.01$ & $0.8 \%$ & $-3.7 \%$ & $-0.7 \%$ \\
6 & $2.40 \pm 0.03$ & $1.3 \%$ & $-4.6 \%$ & $-1.7 \%$ \\
7 & $2.51 \pm 0.03$ & $1.1 \%$ & $-4.3 \%$ & $-1.1 \%$ \\
& & & & $0.7 \%$
\end{tabular}

measurement at all concentrations, i.e. eqn (1) can be simplified to eqn (3).

Gradient-free CIGSe reference samples with different CGI and GGI were grown and characterized in order to test these two conditions for $\mathrm{Cu}$, Se, In, and $\mathrm{Ga}$. Additional calibration samples were used to do so for S and Na. In Fig. 2(a) to (f) the calibration curves of the used elemental transitions of $\mathrm{Cu}, \mathrm{Se}$, $\mathrm{In}, \mathrm{Ga}, \mathrm{S}$, and $\mathrm{Na}$ are displayed. Note that we distinguish between reference samples, which are used for both the calibration curves and the quantification of the unknown samples and therefore have the same matrix and similar composition to the latter (marked green in Fig. 2) as well as calibration samples (marked black), which are only used to generate calibration curves over a wider range. Excluding thus background and non-linearity issues, the validity of the quantification algorithm described above using just the matrix specific samples (green symbols) and no background correction is confirmed.

As can be seen in Fig. 2(a)-(d), the calibration curves of the used emission lines of $\mathrm{Cu}$, Se, In, and Ga (Cu: $327 \mathrm{~nm}$, In: $451 \mathrm{~nm}$, Ga: $417 \mathrm{~nm}$, Se: $196 \mathrm{~nm}$ ) are linear and don't show any significant background at $c_{\mathrm{j}} q=0$. Therefore we can safely neglect $S_{\mathrm{j}}$ and $b_{\mathrm{j}}$ in these cases. The emission lines of $\mathrm{S}$ at $181 \mathrm{~nm}$ and the one of $\mathrm{Na}$ at $589 \mathrm{~nm}$, however, show a more complex behaviour. The S-line does show a linear dependence on $c_{\mathrm{S}} q$, but also shows a background at $c_{\mathrm{S}} \cdot q=0$ of about 0.007 . While this background is negligible in the case of most CIGSSe-samples (CIGSSe-samples grown in our lab exhibit S-concentrations lying within the yellow marked area in Fig. 2(e)), one has to be careful when analysing Sfree samples. In the latter case one has to either exclude the Ssignal completely from the quantification, which seems reasonable if there is no risk of contamination, or to manually subtract the background from the measured S-signal.

The Na-line on the other hand is not affected by any background, but shows a non-linear dependence of the measured intensity on $c_{\mathrm{Na}} q$. This saturation of the intensity at high Na-concentration is most likely due to self-absorption occurring for the used emission line. This means that photons, which are emitted by Na-atoms sputtered from the sample, don't reach the detector, because they are absorbed by Na-atoms in the region between the plasma and source window. Since this effect is exponentially dependent on the number of atoms in the volume of absorption, ${ }^{35}$ it becomes more important at higher concentrations and sputtering rates. If an emission line is strongly affected by self-absorption (this is often the case for emission lines to the ground state at so-called resonance lines) the influence of the selfabsorption can also lead to a change of the line-shape of the measured peak, which has to be taken into account as well. The reader is referred to ref. 35 for further details on this issue.

However, since the data can be interpolated by a linear fit function for Na-concentrations typically found in CIGSSe solar cells $\left(<1 \times 10^{-3}\right)$ with a reasonable error (see comparison of the fitting curves in Fig. 2(f)), $S_{\mathrm{j}}$ can still be neglected in our case. If analysing samples with high Na-concentrations, one would have to fit a quadratic or higher order polynomial function to 

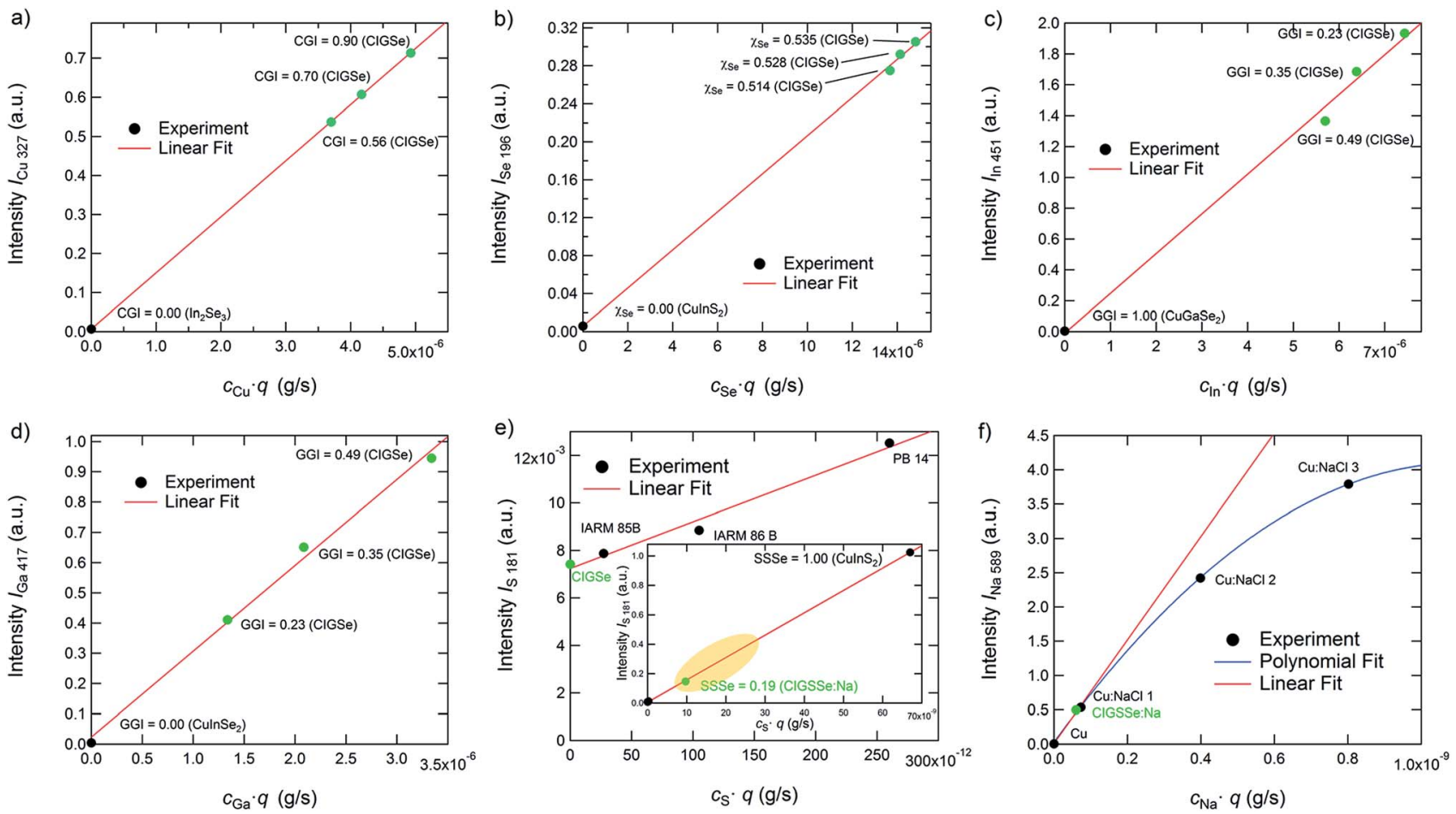

Fig. 2 Calibration curves of (a) Cu, (b) Se, (c) In, (d) Ga, (e) S, and (f) Na. Black symbols mark samples, which were only used for these calibration curves, while samples marked in green are additionally used as reference samples during the quantification.

quantify the Na-signal. Please note that the dependences shown in this section are only valid for the used system and sputtering parameters.

\section{Reference samples}

In order to be a suitable reference sample for a matrix specific determination of the emission yields, the sample has to meet several requirements:

(1) Similar matrix to the unknown samples.

(2) Vacuum tightness to act as a sealing against the atmosphere and stability during the sputtering process.

(3) Known concentrations $c_{\mathrm{j}}$ of all contained elements.

(4) Known sputtering rate.

If the layers are used for calibration:

(5) Known thickness of the complete layer.

(6) Homogeneous distribution of all contained elements throughout the layer.

(7) Known density of the layer.

Requirements (1), (2) and to (6) can be easily met in the case of S-free CIGSe, since gradient-free CIGSe layers can be grown in a co-evaporation system. The $c_{\mathrm{j}}$ of all contained elements and the thickness of the layer are adjusted with in situ optical process controls and are verified by XRF. ${ }^{36,37}$ The density of the layers is determined using eqn (14).

Since our co-evaporation system is not equipped with an Ssource, we cannot grow reference samples for CIGSSe ourselves. Therefore a cold-pressed CIGSSe:Na sample meeting all the requirements listed above was included. In Fig. SI 2 of the ESI $\dagger$ images of both a self-grown CIGSe and the CIGSSe:Na reference sample and the corresponding qualitative depth profiles are shown.

Since the CIGSSe:Na sample is about $2 \mathrm{~cm}$ thick, the reference measurement cannot be performed through the whole sample but has to be stopped manually. The depth of the sputtered crater has to be determined in an extra step. This can be done directly by measuring it with a profilometer or indirectly by comparing the emission yields of $\mathrm{Cu}$, In, $\mathrm{Ga}$ and $\mathrm{Se}$ with the ones obtained from the thin film reference. In the latter case, the sputtering depth of the CIGSSe:Na sample is used as a free parameter to correlate the emission yields of $\mathrm{Cu}, \mathrm{In}, \mathrm{Ga}$ and Se measured in the CIGSSe:Na reference with the ones obtained from the thin film sample with known thickness. The result of this fit is used for the determination of the emission yields of $\mathrm{S}$ and $\mathrm{Na}$. $\mathrm{Na}$ is the main dopant in CIGSSe absorber layers ${ }^{38}$ and its depth profile can give valuable information about diffusion processes during the formation of the absorber layer. The importance of the ability to measure the Na depth profile is discussed below.

\section{Examples of applications}

Determination of the Bandgap Energy Profiles. There have been several empirical formulae derived to calculate the bandgap energy from the elemental ratios. In this work we use the formula established by Ishizuka et al. ${ }^{39}$ for the calculation of the $E_{\mathrm{g}}$-profile from the GGI in S-free CIGSe absorber layers:

$$
E_{\mathrm{g}}=1+0.564 \times \mathrm{GGI}+0.116 \times \mathrm{GGI}^{2} .
$$


In the case of CIGSSe absorber layers containing $\mathrm{S}$ we use the formula published by Bär et al.:40

$$
\begin{aligned}
E_{\mathrm{g}}= & +0.13 \times \mathrm{GGI}^{2}+0.08 \times \mathrm{GGI}^{2} \cdot \mathrm{SSSe} \\
& +0.13 \times \mathrm{GGI} \cdot \mathrm{SSSe}+0.55 \times \mathrm{GGI}+0.54 \mathrm{SSSe} .
\end{aligned}
$$

Bandgap-profiling of co-evaporated $\mathrm{Cu}(\mathrm{In}, \mathrm{Ga}) \mathrm{Se}_{2}$. In Fig. 3(a) the quantified depth profile of a standard CIGSe absorber layer is shown. GGI and CGI as well as the $E_{\mathrm{g}}$-profile can be derived from the elemental composition (see Fig. 3(b)). The minimum $E_{\mathrm{g}}$ is derived as $E_{\mathrm{g}, \min }=(1.12 \pm 0.02) \mathrm{eV}$ and increases from there towards both the front and the back interface. In Fig. 4 the derivative of the EQE of the corresponding solar cell is shown. The EQE can be interpreted as a wavelength-dependent measure of how many electrons contribute to the photocurrent per incoming photon. ${ }^{\mathbf{4 1}}$ Therefore the inflection point of the strong increase of the EQE-signal, i.e. the maximum of the derivative of EQE, can be used as a measure of the optical bandgap energy of the solar cell, which is the minimum of the $E_{\mathrm{g}}$-profile. The optical bandgap energy is $E_{\mathrm{g}, \min }=(1.13 \pm 0.01) \mathrm{eV}$. This value is in excellent agreement with the one derived from GD-OES and shows that the quantification of the GD-OES measurements is

a)

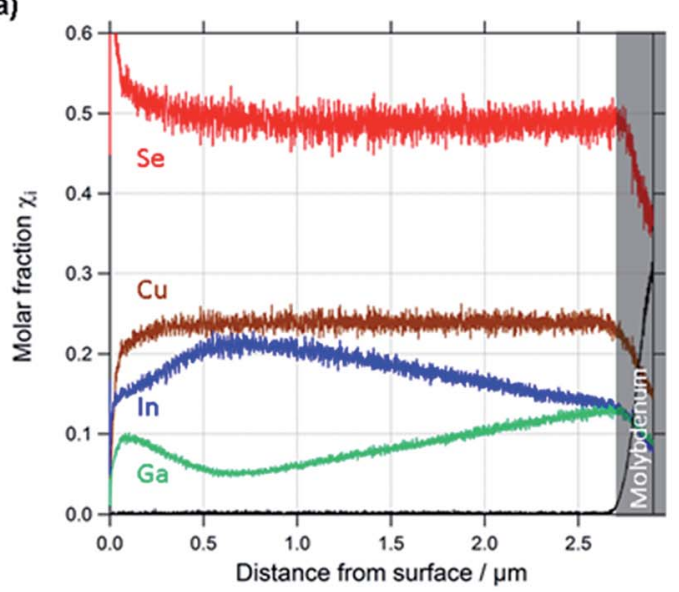

b)

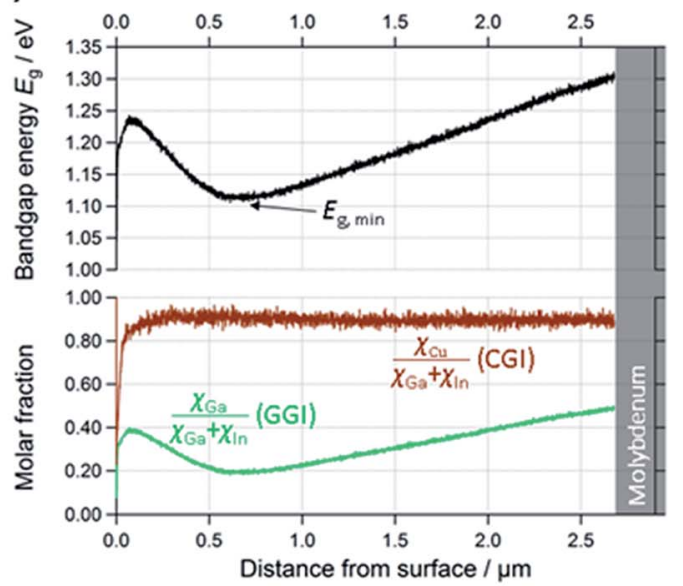

Fig. 3 (a) Quantified depth profile of a standard CIGSe layer. (b) Calculated profiles of CGl, GGl and $E_{\mathrm{g}}$ of the same layer.

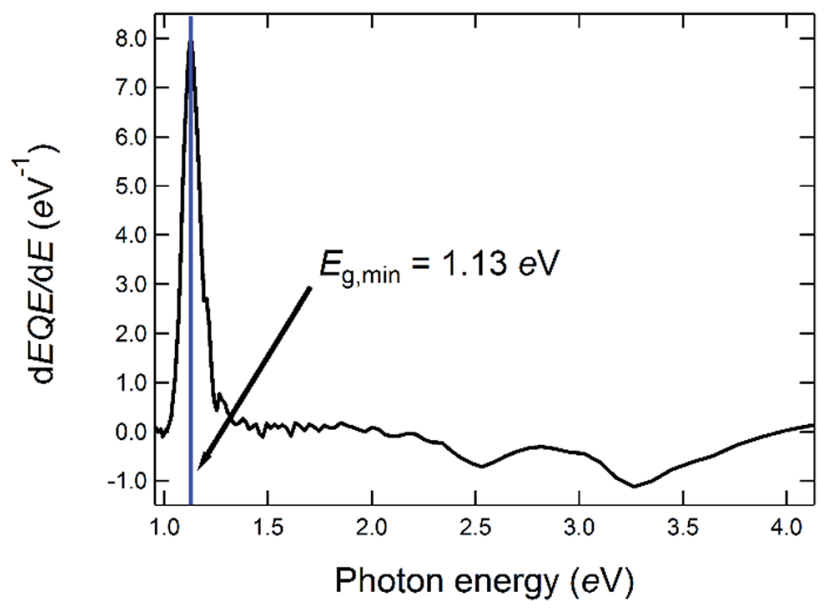

Fig. 4 Determination of the optical bandgap energy from the first derivative of the EQE of a solar cell made from a CIGSe-absorber from the same deposition run as the one for which the GD-OES profile is shown in Fig. 3.
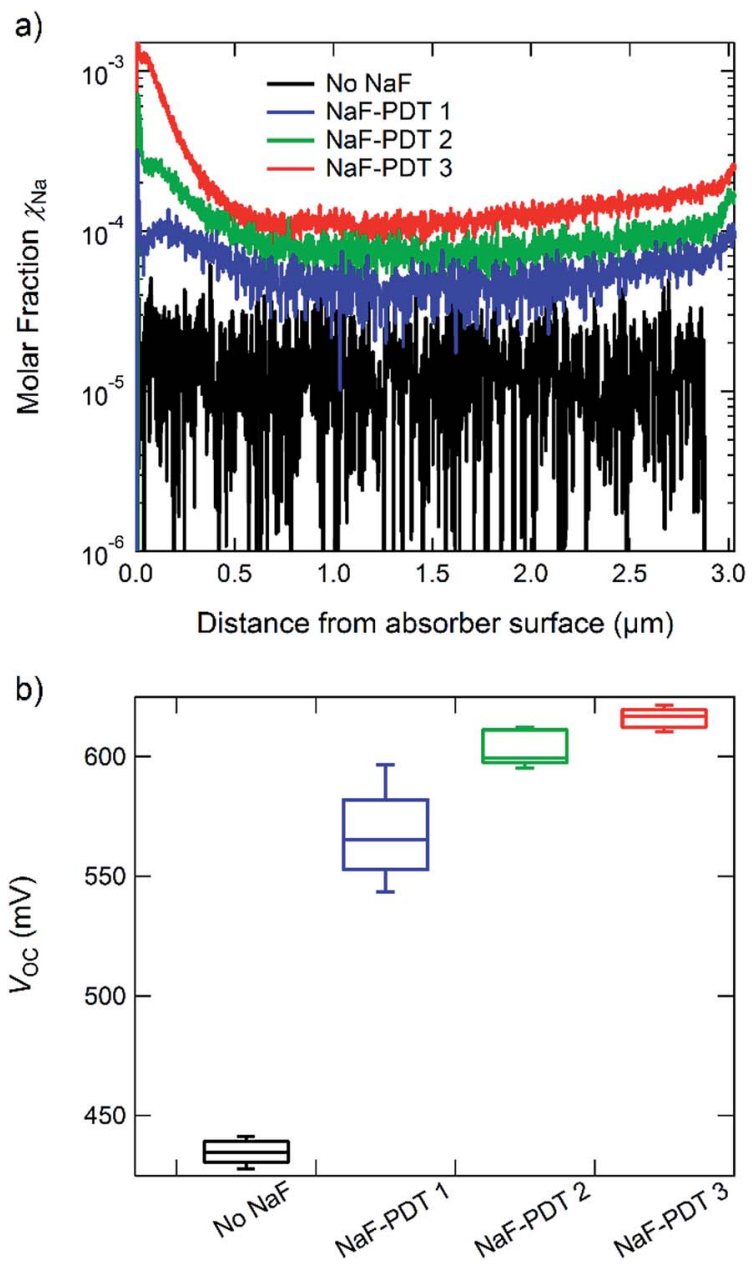

Fig. 5 (a) Molar fraction of $\mathrm{Na}$ of four different samples with different amounts of $\mathrm{Na}$ incorporated derived from GD-OES measurements. (b) Open-circuit voltage of the corresponding solar cells. 
accurate for CIGSe-devices with standard Cu-composition (CGI in the range of 0.9 ).

Bandgap profiling of sequentially processed $\mathrm{Cu}(\mathrm{In}, \mathrm{Ga})(\mathrm{S}, \mathrm{Se})_{2}$. As already shown in a previous publication, ${ }^{\mathbf{4 2}}$ an evaluation of the quantification of GD-OES depth profiles of S containing CIGSSe is more challenging. In that publication we showed that the S-distribution in sequentially processed CIGSSe absorber layers is strongly inhomogeneous at a microscopic level (fluctuations in a range of $1 \mu \mathrm{m}$ ). It is also shown that $\mathrm{S}$ is not incorporated into most of the grains but is located at grain boundaries, interfaces and surfaces. Since GD-OES with a 2.5 $\mathrm{mm}$ anode is laterally integrated over an area of about $5 \mathrm{~mm}^{2}$, it is not sensitive to these fluctuations. Therefore the minimum $E_{\mathrm{g}}$ as measured by GD-OES in this kind of absorber layer does not correspond to the actual optical active bandgap energy. However, it can still show the S-distribution at a macroscopic level and therefore in comparison with EQEmeasurements be used as a quick and easily accessible tool for the optimization of the process parameters in such a sulfurization process.

Determination of the Na-distribution within Co-evaporated $\mathbf{C u}(\mathbf{I n}, \mathbf{G a}) S_{2}$. In Fig. 5(a) the Na-depth profiles of four samples with different amounts of $\mathrm{Na}$ incorporated into the CIGSe absorber layer are shown. All samples were prepared with a $\mathrm{SiO}_{x} \mathrm{~N}_{y}$-barrier which hinders Na-diffusion from the glass. For three of the samples NaF was deposited onto the finished CIGS absorber layer subsequently to the growth. From "NaF-PDT 1" to "NaF PDT 3" the source temperature of the NaF-crucible was steadily increased, while all other parameters of the deposition were kept constant. The GD-OES profiles shown in Fig. 5(a), which were measured after rinsing the samples in $\mathrm{NH}_{4} \mathrm{OH}$, show that a higher source temperature actually leads to a higher amount of $\mathrm{Na}$ incorporated into the films. In Fig. 5(b) the open circuit voltage $\left(V_{\mathrm{OC}}\right)$ measured for the corresponding solar cells is displayed. As expected, ${ }^{38} V_{\mathrm{OC}}$ increases with the amount of $\mathrm{Na}$ incorporated into the CIGSe validating the results of the GDOES measurements. Since the detection of Na by XRF can be challenging, GD-OES is one of the easiest and fastest ways to accurately determine its concentration.

\section{Conclusions}

In the present contribution we showed how to optimize the sputtering parameters of pulsed GD-OES for investigating the elemental depth profiles of CIGSSe thin films. After ensuring optimum sputtering conditions, one emission line for each contained element was tested for its suitability for quantitative depth profiling. We could demonstrate for the first time that the utilized emission lines of $\mathrm{Cu}$, In, $\mathrm{Ga}$ and Se exhibit a linear dependence on the product of concentration times and sputtering rate and are therefore indeed usable for the quantification. Furthermore we demonstrated that the suitability of the emission lines of $\mathrm{S}$ and $\mathrm{Na}$ used here has to be carefully evaluated for each specific case and hence further corrections might be necessary. A quantification algorithm based on the assumption of constant emission yield is applied to the case of
CIGSSe. The accuracy of the final quantified profiles of the molar fractions of each element was demonstrated by several examples. These results subsequently justify the use of GD-OES in general and the tested emission lines in particular for quantitative compositional depth profiling of CIGSSe thin films.

\section{Conflicts of interest}

There are no conflicts to declare.

\section{Acknowledgements}

The authors thank S. Cinque, S. Falk and J. Lauche for maintaining the GD-OES system, V. Hinrichs for performing the LAICP-MS measurements, C. Stephan for fruitful discussions as well as B. Bunn, M. Hartig, J. Lauche and T. Münchenberg for preparation of the substrates and absorber layers. They also thank K. Coulibaly for profilometer measurements, H. Merker and S. Donath for the preparation of sintered calibration samples, A. Lomuscio from the University of Luxembourg for the preparation of the $\mathrm{CuInS}_{2}$-thin film and C. Prinz from the Bundesanstalt fuer Materialforschung und -pruefung (BAM) for measurements of the sample density.

\section{References}

1 M. A. Green, Y. Hishikawa, E. D. Dunlop, D. H. Levi, J. HohlEbinger and A. W. Y. Ho-Baillie, Prog. Photovoltaics, 2018, 26, 3-12.

2 T. Kato, J.-L. Wu, Y. Hirai, H. Sugimoto and V. Bermudez, IEEE J. Photovolt., 2018, 1-6.

3 W. Shockley and H. J. Queisser, J. Appl. Phys., 1961, 32, 510519.

4 M. A. Contreras, J. Tuttle, A. M. Gabor, A. Tennant, K. Ramanathan, S. Asher, A. Franz, J. Keane, L. Wang, J. Scofield and R. Noufi, Proc. 1st WCPEC, Waikoloa, Hawaii, 1994, pp. 83-86.

5 S.-H. Wei and A. Zunger, J. Appl. Phys., 1995, 78, 3846-3856. 6 L. M. Peter, Philos. Trans. R. Soc., A, 2011, 369, 1840-1856.

7 T. M. Friedlmeier, P. Jackson, A. Bauer, D. Hariskos, O. Kiowski, R. Menner, R. Wuerz and M. Powalla, Thin Solid Films, 2017, 633, 13-17.

8 T. Dullweber, O. Lundberg, J. Malmström, M. Bodegard, L. Stolt, U. Rau, H. W. Schock and J. H. Werner, Thin Solid Films, 2001, 387, 11-13.

9 A. M. Gabor, J. R. Tuttle, D. S. Albin, M. A. Contreras, R. Noufi and A. M. Hermann, Appl. Phys. Lett., 1994, 65, 198-200.

10 D. Abou-Ras, R. Caballero, C.-H. Fischer, C. A. Kaufmann, I. Lauermann, R. Mainz, H. Mönig, A. Schöpke, C. Stephan, C. Streeck, S. Schorr, A. Eicke, M. Döbeli, B. Gade, J. Hinrichs, T. Nunney, H. Dijkstra, V. Hoffmann, D. Klemm, V. Efimova, A. Bergmaier, G. Dollinger, T. Wirth, W. Unger, A. A. Rockett, A. Perez-Rodriguez, J. Alvarez-Garcia, V. Izquierdo-Roca, T. Schmid, P.-P. Choi, M. Müller, F. Bertram, J. Christen, H. Khatri, R. W. Collins, 
S. Marsillac and I. Kötschau, Microsc. Microanal., 2011, 17, 728-751.

11 T. Delsol, A. P. Samantilleke, N. B. Chaure, P. H. Gardiner, M. Simmonds and I. M. Dharmadasa, Sol. Energy Mater. Sol. Cells, 2004, 82, 587-599.

12 R. Caballero, C. A. Kaufmann, V. Efimova, T. Rissom, V. Hoffmann and H.-W. Schock, Prog. Photovoltaics, 2011, 21, 30-46.

13 M. Powalla, P. Jackson, W. Witte, D. Hariskos, S. Paetel, C. Tschamber and W. Wischmann, Sol. Energy Mater. Sol. Cells, 2013, 119, 51-58.

14 T. Klinkert, M. Jubault, F. Donsanti, D. Lincot and J.-F. Guillemoles, Thin Solid Films, 2014, 558, 47-53.

15 D. Mercier, M. Bouttemy, J. Vigneron, P. Chapon and A. Etcheberry, Appl. Surf. Sci., 2015, 347, 799-807.

16 P. Haunault, C. Morin, and P. Chapon, IEEE $42^{\text {nd }}$ PVSC, New Orleans, Louisiana, 2015, DOI: 10.1109/PVSC.2015.7356028.

17 S. Zahedi-Azad and R. Scheer, Phys. Status Solidi C, 2017, 14, 1600203.

18 W. Zhang, H. Zhu, L. Zhang, Y. Guo, X. Niu, Z. Li, J. Chen, Q. Liu and Y. Mai, Solid-State Electron., 2017, 132, 57-63.

19 A. Bengtson and T. Nelis, Anal. Bioanal. Chem., 2006, 385, 568-585.

20 M. D. Heinemann, R. Mainz, F. Österle, H. RodriguezAlvarez, D. Greiner, C. A. Kaufmann and T. Unold, Sci. Rep., 2017, 7, 45463.

21 A. Lomuscio, M. Melchiorre and S. Siebentritt, IEEE 7th WCPEC, 2018, DOI: 10.1109/PVSC.2018.8548252.

22 C. Gonzalez-Gago, P. Smid, T. Hofmann, C. Venzago, V. Hoffmann and W. Gruner, Anal. Bioanal. Chem., 2014, 406, 7473-7482.

23 S. S. Schmidt, C. Wolf, H. Rodriguez-Alvarez, J.-P. Bäcker, C. A. Kaufmann, S. Merdes, F. Ziem, M. Hartig, S. Cinque, I. Dorbandt, C. Köble, D. Abou-Ras, R. Mainz and R. Schlatmann, Prog. Photovoltaics, 2017, 25, 341-357.

24 W. Grimm, German Patent 1, 589 389, 1967.

25 V. Efimova, V. Hoffmann and J. Eckert, J. Anal. At. Spectrom., 2011, 26, 784-791.
26 L. Wilken, V. Hoffmann, H.-J. Uhlemann, H. Siegel and K. Wetzig, J. Anal. At. Spectrom., 2003, 18, 646-655.

27 V. Efimova, PhD thesis, Technical University Dresden, 2011. 28 R. Payling, Glow Discharge Optical Emission Spectrometry, John Wiley \& Sons, Hoboken, New Jersey, 1997, ch. 6.4, pp. 287-291.

29 V. Efimova, V. Hoffmann and J. Eckert, Spectrochim. Acta, Part B, 2012, 76, 181-189.

30 D. Abou-Ras, B. Marsen, T. Rissom, F. Frost, H. Schulz, F. Bauer, V. Efimova, V. Hoffmann and A. Eicke, Micron, 2012, 43, 470-474.

31 L. Wilken, V. Hoffmann and K. Wetzig, J. Anal. At. Spectrom., 2003, 18, 1141-1145.

32 L. Vegard, Z. Phys., 1921, 5, 17-26.

33 C. Stephan, S. Schorr, M. Tovar and H.-W. Schock, Appl. Phys. Lett., 2011, 98, 091906.

34 C. Stephan, D. Greiner, S. Schorr and C. A. Kaufmann, J. Phys. Chem. Solids, 2016, 98, 309-315.

35 R. D. Cowan and G. H. Dieke, Rev. Mod. Phys., 1948, 20, 418456.

36 R. Scheer, A. Neisser, K. Sakurai, P. Fons and S. Niki, Appl. Phys. Lett., 2003, 82, 2091-2093.

37 R. Scheer, A. Pérez-Rodriguez and W. K. Metzger, Prog. Photovoltaics, 2010, 18, 467-480.

38 D. Rudmann, PhD thesis, ETH Zürich, 2004.

39 S. Ishizuka, K. Sakurai, A. Yamada, H. Shibata, K. Matsubara, M. Yonemura, S. Nakamura, H. Nakanishi, T. Kojima and S. Niki, Jpn. J. Appl. Phys., 2005, 44, 679-682.

40 M. Bär, W. Bohne, J. Röhrich, E. Strub, S. Lindner, M. C. LuxSteiner, Ch.-H. Fischer, T. P. Niesen and F. Karg, J. Appl. Phys., 2004, 96, 3587-3590.

41 S. Siebentritt, Sol. Energy Mater. Sol. Cells, 2011, 95, 14711476.

42 T. Kodalle, S. S. Schmidt, C. Wolf, D. Greiner, U. Bloeck, P. Schubert-Bischoff, C. A. Kaufmann and R. Schlatmann, J. Alloys Compd., 2017, 703, 600-604. 